\title{
BMJ Open Study protocol of the global Effisayil 1 Phase II, multicentre, randomised, double-blind, placebo-controlled trial of spesolimab in patients with generalized pustular psoriasis presenting with an acute flare
}

Siew Eng Choon, ${ }^{1}$ Mark G Lebwohl, ${ }^{2}$ Slaheddine Marrakchi, ${ }^{3}$ A David Burden, ${ }^{4}$ Tsen-Fang Tsai, ${ }^{5}$ Akimichi Morita, ${ }^{6}$ Alexander A Navarini, ${ }^{7}$ Min Zheng, ${ }^{8}$ Jinhua Xu, ${ }^{9}$ Hamida Turki, ${ }^{3}$ Sushmita Rajeswari, ${ }^{10}$ Hongjie Deng, ${ }^{11}$ Kay Tetzlaff, ${ }^{12}$ Christian Thoma, ${ }^{13}$ Hervé Bachelez ${ }^{14,15}$

To cite: Choon SE, Lebwohl MG, Marrakchi S, et al. Study protocol of the global Effisayil 1 Phase II, multicentre, randomised, double-blind, placebo-controlled trial of spesolimab in patients with generalized pustular psoriasis presenting with an acute flare. BMJ Open 2021;11:e043666. doi:10.1136/ bmjopen-2020-043666

- Prepublication history and additional material for this paper is available online. To view these files, please visit the journal online (http://dx.doi.org/10. 1136/bmjopen-2020-043666).

Received 10 August 2020 Revised 14 January 2021 Accepted 02 February 2021

Check for updates

(C) Author(s) (or their employer(s)) 2021. Re-use permitted under CC BY-NC. No commercial re-use. See rights and permissions. Published by BMJ.

For numbered affiliations see end of article.

Correspondence to

Dr Hervé Bachelez;

herve.bachelez@aphp.fr

\section{ABSTRACT}

Introduction Generalized pustular psoriasis (GPP) is a rare, potentially life-threatening disease characterised by recurrent flares of widespread neutrophilic aseptic skin pustular eruption. Despite the availability of approved biologics for GPP in Japan, Taiwan and Thailand, associated evidence is largely based on uncontrolled studies in which acute flares were not directly assessed. Therefore, there is a high unmet need to investigate new rapid-acting effective treatments that resolve symptoms associated with acute GPP flares. A prior Phase I proofof-concept study showed rapid improvements in skin and pustule clearance with a single intravenous dose of spesolimab, a novel anti-interleukin-36 receptor antibody, in patients presenting with an acute GPP flare. Here, we present the design and rationale of Effisayil 1, a global, Phase II, placebo-controlled study to evaluate the efficacy, safety and tolerability of spesolimab in patients presenting with an acute GPP flare.

Methods and analysis At least 51 patients with an acute GPP flare will be randomised 2:1 to receive a single $900 \mathrm{mg}$ intravenous dose of spesolimab or placebo and followed for up to 28 weeks. The primary endpoint is a Generalized Pustular Psoriasis Physician Global Assessment (GPPGA) pustulation subscore of 0 (pustule clearance) at Week 1 . The key secondary endpoint is a GPPGA score of 0 or 1 (clear or almost clear) at Week 1. Safety will be assessed over the study duration by the occurrence of treatment-emergent adverse events. Blood and skin biopsies will be collected to assess biomarkers. Superiority of spesolimab over placebo in the proportion of patients achieving the primary and key secondary endpoints will be evaluated.

Ethics and dissemination The study complies with the ethical principles of the Declaration of Helsinki, the International Council for Harmonisation's Good Clinical Practice and local regulations. Ethics committee approvals have been obtained for each centre from all participating countries and are listed in online supplementary file 1.
Strengths and limitations of this study

To our knowledge, this is the first randomised, double-blind, placebo-controlled study in patients presenting with an acute generalized pustular psoriasis (GPP) flare.

- This study will be the largest randomised, placebocontrolled trial conducted in this population to date.

- This study will incorporate clinically relevant disease-specific measures to assess the efficacy of spesolimab, an anti-interleukin-36 receptor antibody for which rapid improvements in skin and pustule clearance has been observed in a previous Phase I single-arm study in seven patients presenting with acute GPP.

- This study will provide robust evidence on the efficacy of spesolimab in patients with acute GPP flares and will allow the natural course of disease to be characterised.

- A major challenge for the study is the recruitment challenges common to rare diseases, which will be minimised by the inclusion of a high number of centres and a more favourable chance of receiving active treatment with a 2:1 allocation ratio.

Primary results will be published in a peer-reviewed journal.

Trial registration details ClinicalTrials.gov identifier: NCT03782792; Pre-results.

\section{INTRODUCTION}

Generalized pustular psoriasis (GPP) is a rare, potentially life-threatening autoinflammatory neutrophilic skin disease characterised by episodes of widespread eruption of aseptic, macroscopically visible pustules, 
which can occur with or without plaque psoriasis, and may be accompanied by systemic inflammation. ${ }^{12}$ GPP is usually associated with one or several systemic symptoms such as fever, malaise and fatigue, and extracutaneous manifestations such as arthritis, uveitis, acute respiratory distress syndrome, cardiovascular shock and neutrophilic cholangitis. ${ }^{34}$ Common laboratory abnormalities include elevated C-reactive protein, leucocytosis, neutrophilia and liver function abnormalities. ${ }^{35}$ Acute GPP flares are associated with significant morbidity, and without appropriate treatment, mortality. ${ }^{4}$ GPP is highly heterogeneous, with some patients experiencing frequent flares, that is, several episodes per year, while for others, flares may occur less frequently, potentially years apart. Acute GPP flares may be triggered by infections, stress, medication, medication withdrawal (eg, corticosteroids) and pregnancy, causing a dramatic reduction in quality of life. ${ }^{4-9}$ During the disease course, some patients with GPP may experience relapsing disease with recurrent flares, or persistent disease with intermittent flares. The clinical appearance of the disease can be phenotypically heterogeneous; skin may be clear in between episodic acute flares or patients may have persistent disease characterised by ill-defined erythematous plaques with or without pustules, which may be localised or widespread. ${ }^{247810-12}$

Therapeutic intervention in GPP is a major challenge globally. The rarity of GPP means recruitment of sufficient patients to conduct large, randomised, controlled trials to robustly investigate the efficacy and safety of therapeutics is a constant challenge. In addition, the intermittent remission and spontaneously self-limiting episodic pustular flares characteristic of GPP make it difficult to assess the efficacy of any intervention in this population. ${ }^{9}$ Therefore, there is still a lack of robust evidence to guide treatment decisions for GPP. Available management guidelines for GPP are widely based on anti-plaque psoriasis strategies, limited case studies and single-arm, open-label studies and generally recommend ciclosporin, retinoids, infliximab and methotrexate as first-line therapies. ${ }^{11}{ }^{13-15}$ Use of conventional systemic therapy may be associated with cumulative toxicities and limited efficacy, making them inappropriate for longterm disease control. ${ }^{11} 1316$ Although there are therapies specifically indicated for GPP approved in Japan, Taiwan and Thailand, there are currently no approved GPP-specific therapies for acute GPP flares globally. In Japan, tumour necrosis factor (TNF)-alpha inhibitors (adalimumab, infliximab and certolizumab pegol), interleukin-(IL-) 17/IL-17 receptor (IL-17R) inhibitors (secukinumab, brodalumab and ixekizumab) and IL-23 inhibitors (risankizumab and guselkumab) are approved for the treatment of patients with GPP who have had an inadequate response to conventional therapy. ${ }^{13}$ 15-30 The approval of TNF inhibitors was based largely on case studies, whereas the approval of IL-17/IL-17R and IL-23 inhibitors was based on prospective, but small-scale, open-label, single-arm, Phase III studies, in which nondisease-specific endpoints, such as any improvement in the Clinical Global Impression index, were used to assess efficacy in Japanese patients presenting with mildto-moderate GPP as per the Japanese Dermatological Association severity score. As systemic and skin manifestations of acute GPP flares may remit within 2 months in some patients, ${ }^{4}$ in most of these trials, clinical assessment of endpoints were conducted at Week 16, and did not measure clinically meaningful aspects such as the rapid improvement or resolution of painful pustules. In Taiwan and Thailand, brodalumab was approved for the treatment of adults with pustular psoriasis who are candidates for systemic therapy, or adults with GPP who have had an inadequate response to conventional therapy, respectively, both based on a Japanese open-label study which included only 12 patients with GPP. ${ }^{31-33}$

Effective treatments with a very rapid onset of action for acute GPP flares that can allow early control of skin inflammation and the prevention of complications, including pustule formation and systemic manifestations, and are tolerable for both short- and long-term treatment strategies are needed. ${ }^{412}$

In patients with GPP, overexpression of IL-36 inflammatory cytokines in skin lesions and loss-of-function mutations in the gene coding for the IL-36 receptor antagonist $(I L 36 R N)$, as well as mutations in other genes connected with the IL-36 pathway (eg, CARD14, AP1S3, SERPINA3), have been identified in genetic studies for some patients, suggesting that the IL-36 pathway may be central to GPP pathogenesis. ${ }^{834-36}$ Reports for the presence of IL36RN mutations in patients with GPP have ranged between $10 \%$ and $82 \%$, and was lower in cases of GPP associated with plaque psoriasis than in those associated with GPP alone. ${ }^{37}$ Moreover, the knockout of the IL-36R in a murine model of deficiency of IL-36R antagonist (DITRA) led to complete resolution of skin inflammation, ${ }^{39}$ making the blockade of IL-36R signalling a novel and appealing targeted therapeutic approach for patients with GPP.

Results of a Phase I, proof-of-concept study, in which the safety and efficacy of a single intravenous dose of spesolimab (BI 655130), an anti-IL-36R humanised monoclonal antibody, was assessed in seven patients with an acute GPP flare, provided the first evidence for targeting the IL-36 pathway. ${ }^{40}$ In this study, spesolimab resulted in rapid (within 7 days) and sustained improvements (up to last assessment at Week 20) in clinical signs and symptoms irrespective of IL36RN mutation, suggesting that IL-36 plays a pathogenic role among patients with GPP with different genetic backgrounds; this was accompanied by rapid downregulation of molecular signatures from the innate immune response, including neutrophilic pathways, and Th1/Th17-mediated inflammation. Four patients $(57.1 \%)$ had mild-to-moderate drug-related adverse events through Week 20, but no severe or serious adverse events were reported. ${ }^{40}$ This study showed that spesolimab is a promising targeted therapy for acute GPP.

Effisayil 1 is a global, Phase II, multicentre, randomised, double-blind, placebo-controlled study of spesolimab in 
patients presenting with an acute GPP flare (ClinicalTrials.gov identifier: NCT03782792). GPP-specific clinical measures that assess key manifestations of the disease, the Generalized Pustular Psoriasis Physician Global Assessment (GPPGA) and GPPGA pustulation subscore, have been established to evaluate treatment efficacy in this study. The GPPGA is a physician-based assessment of the severity of pustules, erythema and scaling of GPP lesions; each component is scored on a 5-point scale, ranging from 0 (clear) to 4 (severe), and the average is calculated (see online supplementary file 2). To differentiate against placebo with a feasible sample size, a stringent primary endpoint was chosen-achievement of a GPPGA pustulation subscore of 0 (complete pustular clearance) at Week 1. The successful early performance of these scores was demonstrated in the Phase I proof-of-concept study. A GPPGA score of 0 or 1 (clear or almost clear skin) was achieved in five of seven patients by Week 1 and in all patients by Week $4 .{ }^{40}$ The acuteness, severity and potentially life-threatening consequences of other autoinflammatory diseases and the effectiveness shown for the blockade of the IL-1 family, such as the achievement of early inactive disease and sustained remission, ${ }^{42}{ }^{43}$ further support the rationale for using an early efficacy endpoint in patients with GPP. The evaluation of non-pustulation components (erythema and scaling) are to be evaluated as part of the key secondary endpoint, the achievement of a total GPPGA score of 0 or 1.

Here, we describe the rationale, study design and methods of Effisayil 1; to our knowledge, this is the first randomised, double-blind, placebo-controlled study in this patient population presenting with an acute GPP flare. This novel and innovative study will inform on the efficacy and safety of targeting the IL-36 pathway in patients with GPP, and will provide insights into the natural progression of untreated GPP disease through the placebo arm, as well as historical clinical data with particular focus on previous occurrence of flares.

\section{METHODS AND ANALYSIS}

\section{Study objectives}

The primary objective of the Effisayil 1 study is to evaluate the efficacy, safety and tolerability of spesolimab versus placebo in patients presenting with an acute GPP flare. Further objectives include the assessment of pharmacokinetics, anti-drug antibodies and pharmacogenomics of spesolimab, and the exploration of biomarkers in acute GPP. In addition, the natural course of GPP in patients receiving placebo, the response of systemic symptoms of GPP flares to spesolimab and the effects of delaying treatment and further dosing with spesolimab in patients with insufficient initial response will also be explored.

\section{Eligibility criteria}

Patients aged 18 to 75 years with GPP, defined by the European Rare And Severe Psoriasis Expert Network (ERASPEN) at screening, ${ }^{1}$ who satisfy the inclusion criteria are allowed to enrol into the study regardless of whether they are experiencing a flare at the time of screening, as patients in remission can be monitored for up to 6 months for their next acute flare. If required, screening and randomisation can occur on the same visit if patients meet the randomisation criteria (laboratory testing to be conducted by a local laboratory in such instances).

Patients will be enrolled if they have previous evidence of fever associated with flares before randomisation, and/ or mild asthenia, and/or myalgia, and/or elevated C-reactive protein, and/or leucocytosis with peripheral blood neutrophilia above the upper limit of normal, and meet one of the following criteria, regardless of IL36RN mutation status:

- Have a GPPGA score of 0 or 1 and a known and documented history of GPP, or

- Are experiencing an acute GPP flare of moderate-tosevere intensity, or

- Are experiencing their first episode of an acute GPP flare of moderate-to-severe intensity; the diagnosis of GPP is to be confirmed retrospectively by a central external expert committee.

Patients eligible for this trial must comply with all of the following inclusion and exclusion criteria at randomisation.

\section{Inclusion criteria}

Patients must be experiencing an acute GPP flare of moderate-to-severe intensity prior to randomisation, defined in the trial as:

- A GPPGA score of $\geq 3$

- New appearance or worsening of existing pustules

- A GPPGA pustulation subscore of $\geq 2$

- $\geq 5 \%$ body surface covered with erythema and the presence of pustules.

\section{Exclusion criteria}

Patients will be excluded if they are presenting with:

- Synovitis-acne-pustulosis-hyperostosis-osteitis syndrome.

- Erythrodermic plaque psoriasis without pustules or with pustules restricted to psoriatic plaques.

- Drug-triggered acute generalized exanthematous pustulosis.

- Immediate life-threatening flare of GPP or requiring intensive care treatment.

- Dose escalation of their maintenance treatment with ciclosporin, retinoids or methotrexate within 2 weeks prior to randomisation.

- Treatment with any drug, including biologics and systemic drugs considered likely to interfere with the safe conduct of the study or any prior exposure to an IL-36R inhibitor.

Full inclusion and exclusion criteria and restricted concomitant medication can be found in the online supplemental file 2 and online supplemental table 1 . 


\section{Randomisation and intervention}

At least 51 patients presenting with an acute GPP flare are to be randomised to receive a single $900 \mathrm{mg}$ intravenous dose of spesolimab or placebo in a 2:1 ratio on Day 1. Study drug is allocated using computerised Interactive Response Technology and patients and investigators involved will remain blinded until after database lock, unless emergency unblinding is required. This allocation ratio will enable more patients with a distressing and potentially life-threatening disease to be on treatment. This design is also likely to be more appealing to patients because evidence has shown that patients prefer to participate in clinical trials where there is a greater likelihood of receiving active treatment. ${ }^{44}$ As required by some regulatory agencies, and based on the rapid onset of response demonstrated in the Phase I, proofof-concept study and the lack of licensed active interventions, the use of a placebo-controlled parallel group was considered most appropriate to evaluate the efficacy and safety of spesolimab in patients with an acute GPP flare. $^{40}$

\section{Study locations and timings}

The study will enrol patients from across 52 centres in 12 countries; it started in March 2019 and it is expected to complete in 2021. After randomisation, patients will be assessed daily until Day 3. Clinical visits on Days 4 to 7 are optional and need not be attended if a patient has already achieved complete pustular clearance (GPPGA pustulation subscore of 0 ). After patients have received a single dose of spesolimab or placebo at Day 1, patients will be followed for 12 to 28 weeks based on the subsequent treatment response (figure 1). Patients who have not received escape treatment, and who have a GPPGA $\geq 2$ and a pustular component of GPPGA $\geq 2$ at Week 1 , will qualify for treatment with an open-label single intravenous dose of $900 \mathrm{mg}$ spesolimab on Day 8. All randomised patients will continue through the subsequent visits until the end of study. Patients who show no flare symptoms of moderate-to-severe intensity at the end of the study and meet clinical criteria for treatment response at Week 12, or at the subsequent visit for patients on rescue treatment with open-label spesolimab (figure 1), will be eligible to enter a 5-year open-label extension study (ClinicalTrials. gov identifier: NCT03886246). Those not qualifying to enter the open-label extension study, will be followed for up to an additional 16 weeks. Clinical response, photographs of skin lesions, physical examination, examination of vital signs, fever assessment and safety laboratory tests are to be undertaken at each visit. Optional skin biopsies will be taken on Days 1 and 8 and Week 8 . Whole blood for RNA sequencing and serum for soluble protein biomarkers are to be sampled prior to dosing, on Days 1 to 3 and Day 8, Week 2, 4 and 12 and at the end of study visit. Importantly, the IL36RN mutation status is to be determined for all patients.

\section{Escape and rescue medication}

If the severity and progression of the disease worsens within the first week after randomisation and requires immediate treatment, the investigator can treat the patient with escape medication, which is the investigator's choice of standard of care (SoC). However, if the disease condition is stable, it is recommended to wait until the primary endpoint visit (Day 8/Week 1) before prescribing a SoC escape medication because there will be an option to administer open-label spesolimab instead at this time. Due to the absence of an approved standard treatment for GPP and a commonly accepted treatment algorithm, patients in this trial are likely to have a heterogeneous pre-treatment history, given that different SoC are available in different countries.

After Week 1, only one rescue dose with open-label spesolimab is permitted if a patient who previously achieved a clinical response (GPPGA 0 or 1 ) experiences recurrence of a GPP flare. Patients who do not achieve a clinical response, but have disease worsening subsequent to Week 1 , can receive an escape treatment chosen by the investigator.

\section{Study endpoints}

The primary endpoint of the study is a GPPGA pustulation subscore of 0 at Week 1 and the key secondary endpoint is a GPPGA score of 0 or 1 at Week 1. Secondary endpoints at Week 4 included in the statistical strategy are a $75 \%$ improvement in the Psoriasis Area and Severity Index for Generalized Pustular Psoriasis (GPPASI 75), change from baseline in pain Visual Analogue Scale (VAS) score, change from baseline in Psoriasis Symptom Scale (PSS) score and change from baseline in Functional Assessment of Chronic Illness Therapy (FACIT)-Fatigue score.

The GPP-specific clinical efficacy endpoints (GPPGA, GPPASI) were created with minimal modification of the PGA and PASI (replacement of the induration component with pustulation), which are widely used and understood clinical instruments by dermatologists, and were created with the help of leading global experts in GPP and psoriasis vulgaris. The proposed primary endpoint of a GPPGA pustulation subscore of 0 (clear) at Week 1 and the key secondary endpoint of a GPPGA score of 0 or 1 at Week 1 are clinically meaningful as pustules are the primary lesion of the disease and reflect the desired rapid pustule clearance and overall improvement in GPP skin symptoms. Other secondary endpoints include the occurrence of treatment-emergent adverse events. At each visit, GPPGA and GPPASI will be measured to assess sustained efficacy (table 1).

At each applicable visit, patients will be asked to complete patient-reported outcomes (PROs) questionnaires. The order of completion for PROs is recommended to be as follows: PSS, Dermatology Life Quality Index (DLQI), pain VAS, FACIT-Fatigue and 5-level EuroQol-5 dimensions (EQ-5D-5L). Correlations between efficacy endpoints and PROs are to be assessed. The full 

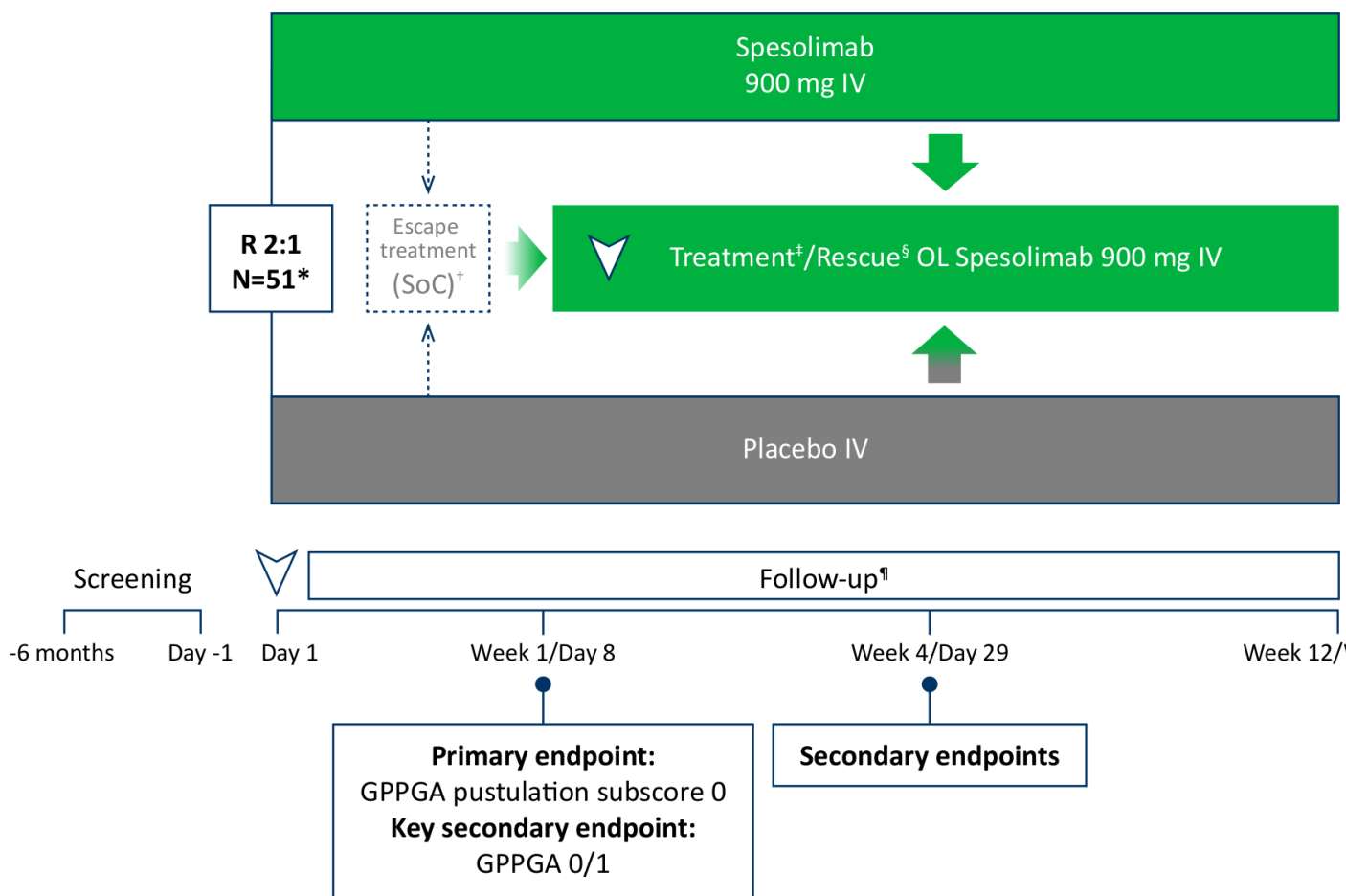

Follow-up"

OLE

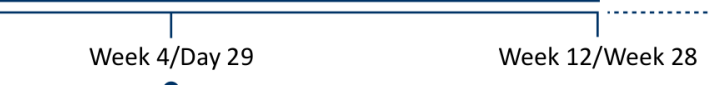

Secondary endpoints

Figure 1 Study design. *Day 1: 51 screened patients with GPP with an acute flare defined as GPPGA $\geq 3$ and GPPGA pustulation subscore of $\geq 2$ will be randomised 2:1 to a single intravenous dose of $900 \mathrm{mg}$ spesolimab or intravenous placebo. ${ }^{\dagger}$ Day 2-7: Escape treatment (SoC) may be offered in case of disease worsening, defined as worsening of clinical status or GPP skin and/or systemic symptoms as defined by the investigator. ${ }^{\ddagger}$ Day 8: Patients with a GPPGA $\geq 2$ and pustular component of GPPGA $\geq 2$ will qualify for treatment with OL spesolimab. ${ }^{\S}$ After Day 8-Week 12: Only one rescue dose with OL spesolimab is permitted if a patient who has previously achieved clinical response (GPPGA 0/1) to initial treatment, either with spesolimab or placebo at Day 1, or escape medication or OL spesolimab at Day 8, experiences a recurrence of a GPP flare ( $\geq 2$-point increase in the GPPGA score and the pustular component of GPPGA $\geq 2$ ). Subsequent flares will be treated with SoC per physician's choice. "Patients who do not require rescue treatment with OL spesolimab are to be followed until Week 12 (EoS) prior to entering into OLE trial. Patients who receive rescue treatment with OL spesolimab between Week 2 and Week 6 are to be followed until Week 12 (EoS) prior to entering the OLE trial. If at Week 12 they qualify to enter the OLE trial, then the EoS will be considered for these patients. If not, patients will have an additional 10 weeks' follow-up and have an EoS at Week 16-28. Patients who receive rescue treatment with OL spesolimab between Weeks 7 and 12 are to be followed for an additional 6 weeks and have a response evaluation at Week 13-18; these patients will not have a visit at Week 12. If at Week 13-18 patients qualify to enter the OLE trial, the EoS will be considered for these patients. If not, patients will have an additional 10 weeks follow-up and have an EoS at Week 16-28. Patients who do not qualify to enter the OLE trial are to be followed for 16 weeks (EoS/Week 16-28) after the last dose of trial medication, which is the latest time point of trial medication given during the study (eg, Day 1, Day 8 if OL spesolimab is given, rescue with OL spesolimab if given). The white arrow head indicates a single dose of intravenous spesolimab or placebo at Day 1 or spesolimab at/after Day 8. EoS, end of study; GPP, generalized pustular psoriasis; GPPGA, Generalized Pustular Psoriasis Physician Global Assessment; IV, intravenous; OL, open label, OLE, open-label extension; R, randomisation; SoC, standard of care.

list of study outcomes is reported in table 1 and online supplemental table 2.

The assessment of biomarkers will be exploratory. This will include biochemical and cellular biomarkers in skin and blood samples pre-treatment and post-treatment with spesolimab. Changes in gene and protein expression in optional skin biopsies, in patients who give consent, are to be assessed. Gene expression analysis will include the genes involved in the mechanism of action of spesolimab or the pathology of the disease. Immunohistochemistry for neutrophils, macrophages, keratinocytes, $\mathrm{T}$ cells and dendritic cells markers are planned. Serum will be collected to assess changes in soluble protein levels of select IL-36 pathway disease-specific biomarkers. Cellular biomarkers on cells such as T cells and macrophages will be assessed by flow cytometry from whole blood samples. In addition, RNA sequencing from one blood sample of IL36RN, CARD14 and AP1S3 genes to assess known GPPassociated mutations will be performed in whole blood, and their potential influence on the severity of disease and efficacy of spesolimab will be evaluated.

\section{Statistical analysis}

The trial is designed to demonstrate the superiority of spesolimab with regard to the primary endpoint (achievement of pustule clearance at Week 1) and the key secondary endpoint (achievement of GPPGA 0 or 1 at Week 1) relative to placebo. With an expected response rate of 0.6 on spesolimab and 0.1 on placebo for the primary endpoint and key secondary endpoint, and a type I error of $<0.025$ (one-sided), for a 
Table 1 Efficacy outcome measures

\begin{tabular}{ll}
\hline Outcome measure & Time point(s) \\
\hline $\begin{array}{l}\text { Primary outcome } \\
\text { GPPGA pustulation subscore of } 0\end{array}$ & Week 1 \\
$\begin{array}{l}\text { Key secondary outcome } \\
\text { GPPGA score of } 0 \text { or } 1\end{array}$ & Week 1 \\
Secondary endpoints & Week 4 \\
GPPASI 75 & Week 4 \\
Change from baseline in VAS score & Week 4 \\
Change from baseline in PSS score & Week 4 \\
Change from baseline in FACIT-Fatigue score & Week 4 \\
GPPGA score of 0 or 1 & Week 4 \\
GPPGA pustulation subscore of 0 & Weeks 1 and 4 \\
GPPASI 50 & Weeks 1 and 4 \\
Percentage reduction from baseline in GPPASI & W
\end{tabular}

Further endpoints to compare the effects of a single intravenous dose of spesolimab to placebo, and/or to explore the effects of OL spesolimab use at Day 8 on the subsequent efficacy of GPP acute flare treatment*

Time to first achievement of a GPPGA score of 0 or 1

Time to first achievement of a GPPGA

pustulation subscore of 0

\begin{tabular}{ll}
\hline Improvement of CGI per JDA severity index & Weeks 1, 2 and 4 \\
\hline GPPGA total score of 0 or $1^{*}$ & By visit \\
\hline GPPGA pustulation subscore of $0^{*}$ & By visit \\
\hline Change from baseline in GPPGA total score & By visit \\
\hline $\begin{array}{l}\text { Change from baseline in GPPGA pustulation } \\
\text { subscore }\end{array}$ & By visit \\
\hline GPPASI $50^{*}$ & By visit \\
\hline GPPASI $75^{*}$ & By visit \\
\hline Overall percent reduction in GPPASI* & By visit \\
\hline Change from baseline in DLQI score & By visit \\
\hline Change from baseline in FACIT-Fatigue score* & By visit \\
\hline Change in pain VAS score* & By visit \\
\hline Change in PSS score* & By visit \\
\hline DLQI score of 0 or 1 & By visit \\
\hline Change from baseline in EQ-5D-5L VAS score & By visit
\end{tabular}

*Endpoints that will also be explored on patients receiving OL spesolimab at Day 8.

CGI, Clinical Global Impression; DLQI, Dermatology Life Quality Index; EQ-5D-5L, 5-level EuroQol-5 dimensions; FACIT, Functional Assessment of Chronic Illness Therapy; GPPASI, Generalized Pustular Psoriasis Area and Severity Index; GPPGA, Generalized Pustular Psoriasis Physician Global Assessment; JDA, Japanese Dermatological Association; OL, open-label; PSS, Psoriasis Symptom Scale; VAS, Visual Analogue Scale.

total sample size of 51 patients, this trial will be able to detect an effect of spesolimab relative to placebo, for the primary endpoint and key secondary endpoint simultaneously, with an overall power of $93.9 \%$. The statistical testing on each of the primary, key secondary and selected secondary endpoints will be performed in a hierarchical manner. The primary endpoint and key secondary endpoint will be analysed with the Suissa-Shuster Z-pooled test to compare the proportion of patients who achieve a response with spesolimab versus placebo at Week 1. For the primary estimand concept, any use of escape medication, open-label spesolimab use at Day 8 or rescue medication with spesolimab, prior to the observation of an endpoint will be considered as non-response. All safety data in this study will be descriptively summarised. For the analysis of biomarkers, a staged approach will be applied, in which the initial analysis will focus on selected time points and decision on further analysis will be made based on these results. Subgroup analysis of trial endpoints on baseline categories (eg, IL36RN mutation status, GPPGA score) are planned. An external and independent data monitoring committee will perform an unblinded safety and efficacy assessment at specified intervals.

\section{Ethics and dissemination}

The study will be conducted in compliance with the protocol, the ethical principles of the Declaration of Helsinki, in accordance with the International Council for Harmonisation's Guideline for Good Clinical Practice (GCP), and the EU regulation 536/2014, the Japanese GCP regulations (Ministry of Health and Welfare Ordinance No. 28, 27 March 1997) and applicable local regulations, and is approved by the ethics committees of participating institutions and countries. A list of all ethical approvals is provided in online supplementary file 1. Approved amendments of the protocol will be posted on ClinicalTrials.gov (last protocol V.3, 26 June 2020). Eligible patients will be provided information and informed consent will be obtained (see online supplementary file 3).

On completion of the trial and after finalisation of the clinical trial report, the study results will be published in an international peer-reviewed medical journal and abstracts for congresses.

\section{Data management}

Patient privacy will be ensured by using patient identification code numbers. Data protection and data security measures are implemented for the collection, storage and processing of patient data in accordance with the confidentiality and privacy principle 12 of the WHO GCP handbook.

\section{Patient and public involvement}

There was no involvement from patients and the public in the design of this study.

\section{DISCUSSION}

Randomised controlled trials are the gold standard for testing the efficacy and safety of new treatments. However, in rare severe diseases, recruitment difficulties and ethical concerns often hamper the possibility of involving a large population in a placebo-controlled randomised trial. Furthermore, although randomised controlled studies have been conducted in rare autoinflammatory syndromes such as cryopyrinopathies with canakinumab, ${ }^{45}$ GPP raised an additional major challenge due to the spontaneously self-limiting course of acute flares in its intermittent pattern that can occur 
in some patients. ${ }^{7}$ Therefore, an original study design integrating these parameters was necessary to accurately assess the efficacy of any drug intervention in this rare variant of the psoriatic disease spectrum. Effisayil 1 is the first randomised, double-blind, placebo-controlled study conducted in patients presenting with an acute GPP flare. Altogether, the high number of participating countries to minimise the risk of under-recruiting, along with the rapidity of the efficacy assessment and the lack of a suitable comparator, propitiates the ambitious design and conduct of this unique trial in a rare disease. This study aims to address a high unmet medical need and the lack of robust efficacy and safety data in patients with acute GPP flares, assess PROs, systemic symptoms and biomarkers and their correlation with clinical response and severity of disease, and provide insights on the natural disease course of an acute GPP flare. Results from this trial are planned to support the first registration of spesolimab in patients with GPP.

The study will evaluate the efficacy and safety of a single intravenous injection of spesolimab at Week 1 versus placebo, with an option of an open-label dose at Day 8 for both treatment arms if criteria are met. In addition, the study will allow the duration of efficacy to be assessed for up to 28 weeks, if not rolling over into the open-label extension study. All recurrent flares within 12 weeks after a single or two intravenous doses of spesolimab will be recorded. Pictures of skin lesions as well as lesion absence will be systematically collected at each visit to provide further visual insights. For each case, naturally occurring resolution or worsening of symptoms in the placebo arm will provide insights on the natural disease course of GPP flare.

Despite the small population size of the study and the single $900 \mathrm{mg}$ dose, Effisayil 1 is designed to be the largest study in patients with GPP, and the first randomised, placebocontrolled trial in this population to date. In addition to this study there are two further studies planned including a 5-year open-label extension study and the Effisayil 2 study (ClinicalTrials.gov identifier: NCT04399837), a multicentre, randomised, parallel-group, double-blind, placebocontrolled, Phase IIb, dose-finding study to evaluate the efficacy and safety of subcutaneous spesolimab compared with placebo in the prevention of GPP flares in patients with a history of GPP. These studies will tackle different disease scenarios that address the limitations of the present study.

Overall, the results of the Effisayil 1 trial will provide robust evidence on early intervention with spesolimab for the treatment of acute GPP flares and will establish the relevance of using disease-specific endpoints that are clinically meaningful for patients and their physicians.

\footnotetext{
Author affiliations

${ }^{1}$ Jeffrey Cheah School of Medicine and Health Sciences, Clinical School Johor

Bahru, Monash University Malaysia, Johor Bahru, Johor, Malaysia

${ }^{2}$ Icahn School of Medicine at Mount Sinai, New York, New York, USA

${ }^{3}$ Dermatology Department, Hedi Chaker University Hospital, Sfax, Tunisia

${ }^{4}$ Institute of Infection, Immunity and Inflammation, University of Glasgow, Glasgow, UK
}

${ }^{5}$ Department of Dermatology, National Taiwan University Hospital and National Taiwan University College of Medicine, Taipei, Taiwan
${ }^{6}$ Department of Geriatric and Environmental Dermatology, Nagoya City University, Graduate School of Medical Sciences, Nagoya, Japan

${ }^{7}$ Department of Dermatology, University Hospital Basel, Basel, Switzerland ${ }^{8}$ Department of Dermatology, Second Affiliated Hospital, Zhejiang University, School of Medicine, Hangzhou, China

${ }^{9}$ Department of Dermatology, Huashan Hospital, Fudan University, Shanghai, China

${ }^{10}$ Boehringer Ingelheim Pharmaceuticals, Ridgefield, Connecticut, USA

${ }^{11}$ Boehringer Ingelheim (China), Investment Co Ltd, Shanghai, China

${ }^{12}$ Boehringer Ingelheim International $\mathrm{GmbH}$, Ingelheim, Germany

${ }^{13}$ Boehringer Ingelheim International GmbH, Biberach, Germany

${ }^{14}$ Service de Dermatologie, Assistance Publique-Hôpitaux de Paris Hôpital SaintLouis, Paris, France

${ }^{15}$ INSERM U1163, Imagine Institute for Genetics of Human Diseases, Université de Paris, Paris, France

Contributors All authors meet the ICMJE criteria for authorship. SEC, MGL, SM, $\mathrm{ADB}, \mathrm{SR}, \mathrm{HD}, \mathrm{CT}$ and $\mathrm{HB}$ were involved in the conception and trial design. $\mathrm{HD}$ provided statistical expertise. SEC, MGL, SM, ADB, TFT, AM, AAN, MZ, JX, HT, SR, HD, $\mathrm{KT}$, CT and $\mathrm{HB}$ contributed in drafting the protocol manuscript and critically revised and commented on its previous versions and the final version. SEC, MGL, SM, ADB, TFT, AM, AAN, MZ, JX, HT, SR, HD, KT, CT and HB will be involved in the analysis and/or interpretation of the data.

Funding This study is funded by Boehringer Ingelheim. Editorial assistance in BM Open and the preparation of this manuscript was provided by Ester Ruiz-Romeu, PhD, Leigh Church, PhD and Tina Borg, PhD, of OPEN Health Communications (London, UK) and funded by Boehringer Ingelheim. The authors would like to acknowledge the contributions made by David Hall, Hairui Hua and Arno Schlosser in the design and conduct of this study. Agreements between Boehringer Ingelheim and the authors included the confidentiality of the study data. All authors collaborated on the writing of the manuscript, and made the decision to submit the manuscript for publication.

Competing interests SR is an employee of Boehringer Ingelheim Pharmaceuticals Inc, Ridgefield, Connecticut, USA. HD is an employee of Boehringer Ingelheim Investment Co Ltd, Shanghai, China. KT is an employee of Boehringer Ingelheim $\mathrm{GmbH}$, Ingelheim, Germany. CT is an employee of Boehringer Ingelheim International $\mathrm{GmbH}$, Biberach, Germany. SEC declares paid activities as advisor, speaker or consultant for AbbVie, Boehringer Ingelheim, Eli Lilly, Janssen, Leo Pharma, MSD, Novartis, Pfizer, Sanofi and UCB. MGL declares paid consulting activities for Aditum Bio, Allergan, Almirall, Arcutis, Inc, Avotres Therapeutics, BirchBioMed Inc, BMD skincare, Boehringer Ingelheim, Bristol Myers Squibb, Cara Therapeutics, Castle Biosciences, Corrona, Dermavant Sciences, Evelo, Facilitate International Dermatologic Education, Foundation for Research and Education in Dermatology, Inozyme Pharma, Kyowa Kirin, LEO Pharma, Meiji Seika Pharma, Menlo, Mitsubishi, NeuroDerm, Pfizer, Promius (Dr Reddy's Laboratories Ltd), Serono, Theravance and Verrica, and research funds from AbbVie, Amgen, Arcutis, Boehringer Ingelheim, Dermavant, Eli Lilly, Incyte, Janssen Research \& Development, LLC, LEO Pharma, Ortho Dermatologics, Pfizer and UCB. SM and HT declare paid consulting activities for Boehringer Ingelheim. ADB declares paid consulting activities for AbbVie, Almirall, Boehringer Ingelheim, Celgene, Janssen, LEO Pharma, Lilly, Novartis and UCB. TFT declares conducting clinical trials or paid consulting activities for AbbVie, Boehringer Ingelheim, Bristol Myers Squibb, Celgene, Eli Lilly, Galderma, GlaxoSmithKline, Janssen-Cilag, LEO Pharma, Merck Sharp \& Dohme, Novartis International, Pfizer and UCB Pharma. AM declares receiving research grants, consulting fees and/or speaker's fees from AbbVie, Boehringer Ingelheim, Celgene, Eli Lilly, Eisai, Janssen, Kyowa Hakko Kirin, LEO Pharma, Maruho, Mitsubishi Tanabe, Nichi-lko, Nippon Kayaku, Novartis, Sun Pharmaceutical Industries, Taiho Pharmaceutical and Torii Pharmaceutical and Ushio. AAN declares being a consultant and advisor and/or receiving speaking fees and/or grants and/or served as an investigator in clinical trials for AbbVie, Almirall, Amgen, BMS, Boehringer Ingelheim, Celgene, Eli Lilly, Galderma, GSK, LEO Pharma, Janssen-Cilag, MSD, Novartis, Pfizer, Sandoz, Sanofi, Serono and UCB. MZ declares receiving grants, consulting fees, and/or speaker's fees from AbbVie, Boehringer Ingelheim, Janssen-Cilag, LEO Pharma China, Novartis, Pfizer Inc and Xian-Janssen. $J X$ declares receiving grants, consulting fees, and/or speaker's fees from AbbVie, Boehringer Ingelheim, Novartis, Pfizer Inc and Sanofi. HB declares paid consulting activities for AbbVie, Almirall, BIOCAD, Boehringer Ingelheim, Celgene, Janssen, Kyowa-Kirin, LEO Pharma, Lilly, Mylan, Novartis and UCB, and grant support from Boehringer Ingelheim, Janssen, LEO Pharma, Novartis and Pfizer.

Patient consent for publication Not required. 
Provenance and peer review Not commissioned; externally peer reviewed.

Supplemental material This content has been supplied by the author(s). It has not been vetted by BMJ Publishing Group Limited (BMJ) and may not have been peer-reviewed. Any opinions or recommendations discussed are solely those of the author(s) and are not endorsed by BMJ. BMJ disclaims all liability and responsibility arising from any reliance placed on the content. Where the content includes any translated material, BMJ does not warrant the accuracy and reliability of the translations (including but not limited to local regulations, clinical guidelines, terminology, drug names and drug dosages), and is not responsible for any error and/or omissions arising from translation and adaptation or otherwise.

Open access This is an open access article distributed in accordance with the Creative Commons Attribution Non Commercial (CC BY-NC 4.0) license, which permits others to distribute, remix, adapt, build upon this work non-commercially, and license their derivative works on different terms, provided the original work is properly cited, appropriate credit is given, any changes made indicated, and the use is non-commercial. See: http://creativecommons.org/licenses/by-nc/4.0/.

\section{REFERENCES}

1 Navarini AA, Burden AD, Capon F, et al. European consensus statement on phenotypes of pustular psoriasis. J Eur Acad Dermatol Venereol 2017;31:1792-9.

2 Ryan TJ, Baker $\mathrm{H}$. The prognosis of generalized pustular psoriasis. Br J Dermatol 1971;85:407-11.

3 Bachelez H. Pustular psoriasis: the dawn of a new era. Acta Derm Venereol 2020;100:87-92.

4 Choon SE, Lai NM, Mohammad NA, et al. Clinical profile, morbidity, and outcome of adult-onset generalized pustular psoriasis: analysis of 102 cases seen in a tertiary hospital in Johor, Malaysia. Int J Dermatol 2014;53:676-84.

5 Viguier M, Allez M, Zagdanski A-M, et al. High frequency of cholestasis in generalized pustular psoriasis: evidence for neutrophilic involvement of the biliary tract. Hepatology 2004;40:452-8.

6 Zelickson BD, Muller SA. Generalized pustular psoriasis. A review of 63 cases. Arch Dermatol 1991;127:1339-45.

7 Baker H, Ryan TJ. Generalized pustular psoriasis. A clinical and epidemiological study of 104 cases. Br J Dermatol 1968;80:771-93.

8 Marrakchi S, Guigue P, Renshaw BR, et al. Interleukin-36-receptor antagonist deficiency and generalized pustular psoriasis. $N$ Engl J Med 2011;365:620-8

9 Bachelez H. Pustular psoriasis and related pustular skin diseases. $\mathrm{Br}$ J Dermatol 2018;178:614-8.

10 Hubler WR. Familial juvenile generalized pustular psoriasis. Arch Dermatol 1984;120:1174-8.

11 Umezawa Y, Ozawa A, Kawasima T, et al. Therapeutic guidelines for the treatment of generalized pustular psoriasis (GPP) based on a proposed classification of disease severity. Arch Dermatol Res 2003;295 Suppl 1:S43-54

12 Boehner A, Navarini AA, Eyerich K. Generalized pustular psoriasis - a model disease for specific targeted immunotherapy, systematic review. Exp Dermatol 2018;27:1067-77.

13 Robinson A, Van Voorhees AS, Hsu S, et al. Treatment of pustular psoriasis: from the Medical Board of the National Psoriasis Foundation. J Am Acad Dermatol 2012;67:279-88.

14 Fujita H, Terui T, Hayama K, et al. Japanese guidelines for the management and treatment of generalized pustular psoriasis: the new pathogenesis and treatment of GPP. J Dermatol 2018;45:1235-70.

15 Imafuku S, Honma M, Okubo Y, et al. Efficacy and safety of secukinumab in patients with generalized pustular psoriasis: a 52week analysis from phase III open-label multicenter Japanese study. J Dermatol 2016;43:1011-7.

16 Iwatsuki K, Terui T, Ozawa A. The guidelines for diagnosis and treatment of generalized pustular psoriasis 2010 (treatment guidelines incorporating TNFa inhibitors). Jpn J Dermatol 2010;120:815-39.

17 Saeki H, Nakagawa H, Ishii T, et al. Efficacy and safety of open-label ixekizumab treatment in Japanese patients with moderate-to-severe plaque psoriasis, erythrodermic psoriasis and generalized pustular psoriasis. J Eur Acad Dermatol Venereol 2015;29:1148-55.

18 Saeki H, Nakagawa H, Nakajo K, et al. Efficacy and safety of ixekizumab treatment for Japanese patients with moderate to severe plaque psoriasis, erythrodermic psoriasis and generalized pustular psoriasis: results from a 52-week, open-label, phase 3 study (UNCOVER-J). J Dermatol 2017;44:355-62.
19 Sano S, Kubo H, Morishima H, et al. Guselkumab, a human interleukin-23 monoclonal antibody in Japanese patients with generalized pustular psoriasis and erythrodermic psoriasis: efficacy and safety analyses of a 52-week, phase 3, multicenter, open-label study. J Dermatol 2018;45:529-39.

20 Yamasaki K, Nakagawa H, Kubo Y, et al. Efficacy and safety of brodalumab in patients with generalized pustular psoriasis and psoriatic erythroderma: results from a 52-week, open-label study. $\mathrm{Br}$ $J$ Dermatol 2017;176:741-51.

21 Wilsmann-Theis D, Schnell LM, Ralser-Isselstein V, et al. Successful treatment with interleukin-17A antagonists of generalized pustular psoriasis in patients without IL36RN mutations. J Dermatol 2018;45:850-4.

22 Fujita H, Terui T, Hayama K, et al. Japanese guidelines for the management and treatment of generalized pustular psoriasis: the new pathogenesis and treatment of GPP. $J$ Dermatol 2018;45:1235-70.

23 Tremfya ${ }^{\circledR}$ (guselkumab), PMDA report; 2018. https://www.pmda.go. jp/files/000234741.pdf

24 Remicade® (infliximab), PMDA report; 2015. https://www.pmda.go. jp/files/000213562.pdf

25 Taltz® (ixekizumab), PMDA report; 2016. https://www.pmda.go.jp/ files/000224346.pdf

26 Lumicef® (brodalumab), PMDA report; 2016. https://www.pmda.go. jp/files/000219040.pdf

27 Cosentyx® (secukinumab), PMDA report; 2015. https://www.pmda go.jp/files/000216877.pdf

28 Eisai Global. Humira® (adalimumab) Approved for pustular psoriasis, 2020. Available: https://www.eisai.com/news/2018/news201824.htm

29 AbbVie News Center. SkyriziтM (risankizumab) Approved for generalized pustular psoriasis, 2020. Available: https://news.abbvie.com/news/ abbvie-announces-first-regulatory-approval-skyrizi-risankizumabfor-treatment-plaque-psoriasis-generalized-pustular-psoriasis-anderythrodermic-psoriasis-and-psoriatic-arthritis-in-japan.htm

30 Cimzia ${ }^{\circledR}$ (certolizumab pegol), PMDA pharmaceuticals and medical devices safety information No. 372; 2020. https://www.pmda.go.jp/ files/000234889.pdf

31 Lumicef® (brodalumab), TFDS assessment report; 2018.

32 Lumicef $®$ (brodalumab), Thailand FDA product information; 2018.

33 Lumicef® (brodalumab), Taiwan CDE report; 2018. https://nihta. cde.org.tw/ReadFile/?p=Assess\&n=ba2820e1-6734-4260-b916f56a3d192e8e.pdf

34 Berki DM, Liu L, Choon S-E, et al. Activating CARD14 mutations are associated with generalized pustular psoriasis but rarely account for familial recurrence in psoriasis vulgaris. J Invest Dermatol 2015;135:2964-70.

35 Setta-Kaffetzi N, Simpson MA, Navarini AA, et al. AP1S3 mutations are associated with pustular psoriasis and impaired Toll-like receptor 3 trafficking. Am J Hum Genet 2014;94:790-7.

36 Frey S, Sticht H, Wilsmann-Theis D, et al. Rare loss-of-function mutation in SERPINA3 in generalized pustular psoriasis. J Invest Dermatol 2020;140:1451-5

37 Johnston A, Xing X, Wolterink L, et al. II-1 and IL-36 are dominant cytokines in generalized pustular psoriasis. J Allergy Clin Immunol 2017;140:109-20.

38 Wang T-S, Chiu H-Y, Hong J-B, et al. Correlation of IL36RN mutation with different clinical features of pustular psoriasis in Chinese patients. Arch Dermatol Res 2016;308:55-63.

39 Blumberg H, Dinh H, Trueblood ES, et al. Opposing activities of two novel members of the IL-1 ligand family regulate skin inflammation. $J$ Exp Med 2007;204:2603-14.

40 Bachelez H, Choon S-E, Marrakchi S, et al. Inhibition of the Interleukin-36 pathway for the treatment of generalized pustular psoriasis. N Engl J Med 2019;380:981-3.

41 Baum P, Visvanathan S, Bossert S, et al. 388 Treatment with spesolimab, an anti-interleukin-36 receptor antibody, in patients with generalized pustular psoriasis, is associated with the downregulation of biomarkers linked to innate, Th1/17 and neutrophilic pathways. $J$ Invest Dermatol 2019;139:S281.

42 Giancane G, Minoia F, Davì S, et al. IL-1 inhibition in systemic juvenile idiopathic arthritis. Front Pharmacol 2016;7:467

43 Federici S, Martini A, Gattorno M. The central role of anti-IL-1 blockade in the treatment of monogenic and multi-factorial autoinflammatory diseases. Front Immunol 2013;4:351.

44 Mills EJ, Seely D, Rachlis B, et al. Barriers to participation in clinical trials of cancer: a meta-analysis and systematic review of patientreported factors. Lancet Oncol 2006;7:141-8.

45 Lachmann HJ, Kone-Paut I, Kuemmerle-Deschner JB, et al. Use of canakinumab in the cryopyrin-associated periodic syndrome. $\mathrm{N} \mathrm{Engl}$ J Med 2009;360:2416-25. 